\title{
COMMENTARY
}

\section{Advancing the science of ventilator-associated pneumonia surveillance}

\author{
Michael Klompas* \\ See related research by Bénet et al., http://ccforum.com/content/16/4/R161
}

\begin{abstract}
The landmark Study on the Efficacy of Nosocomial Infection Control definitively demonstrated that infection surveillance and control programs prevent hospital-acquired infections. The rise of public reporting, benchmarking, and pay for performance movements, however, has considerably changed the infection surveillance landscape in the 27 years since this study was published. Clinically nuanced surveillance definitions that served the profession well for many years have fallen into disfavor because their complexity and subjectivity allow for conscious and subconscious gaming. These limitations make it very difficult to determine whether changes in surveillance rates represent true changes in disease incidence or artifacts of definition subjectivity, external reporting pressures, and internal biases. Surveillance definitions need to be revised to enhance objectivity and to ensure that they detect clinically meaningful events associated with compromised outcomes. The US Centers for Disease Control and Prevention recently released modified definitions for ventilator-associated events that have the potential to make safety surveillance for ventilated patients more credible and useful once again.
\end{abstract}

The report by Bénet and colleagues in the previous issue of Critical Care is at once a welcome reminder of the pivotal importance of infection surveillance programs and a window into current weaknesses in surveillance science that threaten the credibility and utility of infection control programs [1]. Bénet and colleagues assessed the impact of a one-year lapse in surveillance in a medical-surgical ICU (surveillance stopped because the

*Correspondence: mklompas@partners.org

Department of Population Medicine, Harvard Medical School and Harvard Pilgrim Health Care Institute, 133 Brookline Avenue, 6th Floor, Boston, MA 02215, USA infection preventionist charged with supporting the unit became burned out - evidence, perhaps, of the underresourcing of infection control programs). Ventilatorassociated pneumonia (VAP) rates rose from 13.4 VAP cases $/ 1,000$ ventilator-days in the 3 years prior to surveillance interruption to 22.9 VAP cases/1,000 ventilatordays in the 3 years following surveillance interruption. In addition, the average duration of mechanical ventilation rose from 7.7 days to 11.3 days $(P=0.007)$ and hospital mortality rose from $13.5 \%$ to $18.8 \%(P=0.028)$. VAP rates and mortality rates were stable, by contrast, in a parallel medical unit that enjoyed continuous surveillance and infection control support throughout the study period.

Bénet and colleagues' findings echo the seminal conclusions of the Study of the Efficacy of Nosocomial Infection Control (SENIC) published by the Centers for Disease Control and Prevention in 1985 [2]. The SENIC project took advantage of a natural experiment: US hospitals began establishing infection control programs of varying intensities starting in the early 1970 s. SENIC investigators reviewed approximately 338,000 patient charts from 338 representative US hospitals and compared hospital-acquired infection rates in 1970 versus those in 1976. The investigators also characterized the intensity of surveillance and infection prevention activities in each hospital and then measured the association of each with changes in infection rates [3]. After adjusting for changes in population risk profiles, average lengths of stay, nurse-to-patient ratios, and other factors, SENIC investigators found that high-intensity infection surveillance and prevention programs reduced hospital infection rates by $32 \%$ while infection rates rose by $18 \%$ in hospitals without infection control programs [2].

The SENIC project cost $\$ 27$ million and took over a decade to complete but it definitively established the importance of infection surveillance and control programs. The infection surveillance and reporting landscape has changed considerably, however, in the 27 years since SENIC was published. Public reporting, benchmarking, and pay for performance movements have focused considerable attention upon hospitals' VAP rates and thereby generated powerful incentives for hospitals to make their 
rates as low as possible [4]. Close evaluation of Bénet and colleagues' study helps illuminate current weaknesses in VAP surveillance science that are beginning to be addressed by the Centers for Disease Control and Prevention.

Bénet and colleagues made a valiant attempt to adjust for changes in patient mix and severity of illness over time, including factors such as age, gender, immunosuppression, antibiotics on ICU admission, and SAPS II score on ICU admission. Nonetheless, the dramatic rise in patients' average duration of mechanical ventilation and mortality following surveillance interruption raises the possibility of important residual differences in patient characteristics that may better account for the observed jump in VAP rates than surveillance interruption alone. Higher VAP rates alone are probably not sufficient to account for the 3.6-day increase in average duration of mechanical ventilation and the $39 \%$ increase in hospital mortality following surveillance interruption since the relative rarity of VAP tends to limit its impact on population-level outcomes. Bénet and colleagues observed a net increase of 9.3 VAP cases/100 patients: the attributable duration of mechanical ventilation for VAP would have to be about 40 days to increase the average duration of mechanical ventilation for the entire population by the observed 3.6 days. This prediction is almost eight times the additive duration of mechanical ventilation typically associated with VAP [5]. Alternatively or additionally, surveillance interruption may have led to a drop in the frequency of preventive care procedures affecting all patients, such as daily sedative interruptions and spontaneous breathing trials that could account for the observed changes in population-level outcomes as well as the increase in VAP rates [6]. Unfortunately, however, Bénet and colleagues did not present data on preventive care patterns so it is not possible to disentangle the relative contributions of changes in case mix, care patterns, and complication rates towards their patients' ultimate outcomes.

Bénet and colleagues' observed rise in VAP rates following surveillance interruption is also complicated by the strong possibility of observer bias. VAP surveillance is highly subjective. The study VAP definition included criteria such as 'lung infiltrates', 'sputum modification', 'suggestive auscultation, 'low oxyhaemoglobin saturation', and 'increased pulmonary oxygen consumption'. VAP definitions typically do not include concrete guidance on interpreting these criteria; rather, surveyors apply the criteria using their own discretion. Given that this was a retrospective study and infection surveyors were not blinded to unit identities, surveyors' conscious or subconscious expectations regarding VAP rates in a unit that foreswore infection surveillance activities for a year may have influenced their interpretations. Furthermore, the surveyor who resumed surveillance in the target unit following interruption was not the same person responsible for surveillance prior to interruption. Different observers' VAP assessments in a common population can vary by a factor of two or more [7]. We cannot know how much the increase in VAP following surveillance interruption was due to the change in surveillance personnel and different subjective assessments (perhaps influenced by subconscious expectations) versus a bona fide rise in invasive pneumonias.

As Bénet and colleagues' study demonstrates, the problems of patient-mix adjustment and observer bias threaten to undermine the credibility of infection surveillance reports $[8,9]$. Cognizant of these concerns, the Centers for Disease Control and Prevention recently proposed a new surveillance paradigm for ventilatorassociated events that is designed to make surveillance objective, more reproducible, and potentially automatable in hospitals with comprehensive electronic health records $[10,11]$. The new paradigm shifts the emphasis of surveillance from pneumonia in particular to ventilatorassociated complications (VACs) in general. VACs are defined quantitatively on the basis of sustained increases in daily minimum positive end-expiratory pressure or fraction of inspired oxygen after at least two calendar days of stable or improving settings. Early work has shown VAC to be an independent predictor of increased length of stay and increased hospital mortality $[12,13]$. Subdefinitions seek to classify VACs into infectionrelated events and possible and probable pneumonias on the basis of temperature changes, white blood cell counts, antibiotic prescribing, quantity of neutrophils on pulmonary specimen Gram stains, and culture results. The new surveillance definitions do not yet incorporate criteria for case-mix adjustments but this has been identified as an area for active research in the future. For the present, however, these definitions have the potential to make surveillance more objective and efficient.

Bénet and colleagues' study is a valuable reminder of the potential risk associated with under-resourcing infection surveillance and control programs. Better surveillance definitions and better case-mix adjustment protocols will help to enhance the credibility and impact of their important message.

\footnotetext{
Abbreviations

SAPS, Simplified Acute Physiology Score; SENIC, Study of the Efficacy of Nosocomial Infection Control; VAC, ventilator-associated complication; VAP, ventilator-associated pneumonia.

Competing interests

The author declares that he has no competing interests.

Published: 31 October 2012

\section{References}

1. Bénet T, Allaouchiche B, Argaud L, Vanhems P: Impact of surveillance of hospital-acquired infections on the incidence of ventilator-associated pneumonia in intensive care units: a quasi-experimental study. Crit Care
} 
2012, 16:R161

2. Haley RW, Culver DH, White JW, Morgan WM, Emori TG, Munn VP, Hooton TM: The efficacy of infection surveillance and control programs in preventing nosocomial infections in US hospitals. Am J Epidemio/ 1985, 121:182-205.

3. Haley RW, Quade D, Freeman HE, Bennett JV: The SENIC Project. Study on the efficacy of nosocomial infection control (SENIC Project). Summary of study design. Am J Epidemio/ 1980, 111:472-485.

4. Klompas M, Platt R: Ventilator-associated pneumonia - the wrong quality measure for benchmarking. Annals Internal Med 2007, 147:803-805.

5. Safdar N, Dezfulian C, Collard HR, Saint S: Clinical and economic consequences of ventilator-associated pneumonia: a systematic review. Crit Care Med 2005, 33:2184-2193.

6. Girard TD, Kress JP, Fuchs BD, Thomason JW, Schweickert WD, Pun BT, Taichman DB, Dunn JG, Pohlman AS, Kinniry PA, Jackson JC, Canonico AE, Light RW, Shintani AK, Thompson JL, Gordon SM, Hall JB, Dittus RS, Bernard GR, Ely EW: Efficacy and safety of a paired sedation and ventilator weaning protocol for mechanically ventilated patients in intensive care (Awakening and Breathing Controlled trial): a randomised controlled trial. Lancet 2008, 371:126-134.

7. Klompas M: Interobserver variability in ventilator-associated pneumonia surveillance. Am J Infect Control 2010, 38:237-239.

8. Klompas M: Eight initiatives that misleadingly lower ventilator-associated pneumonia rates. Am J Infect Control 2012, 40:408-410.
9. Klompas M: Is a ventilator-associated pneumonia rate of zero really possible? Curr Opin Infect Dis 2012, 25:176-182.

10. Improving Surveillance for Ventilator-associated Events in Adults [http://www.cdc.gov/nhsn/psc_da-vae.html]

11. Magill SS, Fridkin SK: Improving surveillance definitions for ventilatorassociated pneumonia in an era of public reporting and performance measurement. Clin Infect Dis 2012, 54:378-380.

12. Klompas M, Khan Y, Kleinman K, Evans RS, Lloyd JF, Stevenson K, Samore M Platt R: Multicenter evaluation of a novel surveillance paradigm for complications of mechanical ventilation. PLOS ONE 2011, 6:e18062.

13. Klompas M, Magill S, Robicsek A, Strymish JM, Kleinman K, Evans RS, Lloyd JF, Khan Y, Yokoe DS, Stevenson K, Samore M, Platt R; CDC Prevention Epicenters Program: Objective surveillance definitions for ventilator-associated pneumonia. Crit Care Med 2012. doi: 10.1097/CCM.0b013e318260c6d9 [Epub ahead of print]

doi:10.1186/cc11656

Cite this article as: Klompas M: Advancing the science of ventilatorassociated pneumonia surveillance. Critical Care 2012, 16:165. 